\title{
Percepção de mães brasileiras e espanholas sobre os problemas de comportamento dos filhos com TDAH
}

\author{
Ana Flávia Lima Teles da Hora \\ Universidade Ceuma \\ Concepción López \\ Universidad de Murcia, Espanha \\ Petruska Oliveira Baptista \\ Simone Souza da Costa Silva \\ Universidade Federal do Pará, PA, Brasil
}

\begin{abstract}
Resumo
Compreendendo a gravidade que os problemas de comportamento podem acarretar no desenvolvimento da criança ao longo da vida, o principal objetivo deste estudo foi comparar a percepção de mães em relação aos problemas de comportamentos listados no CBCL (Child Behavior Checklist) em duas amostras clínicas compostas de crianças brasileiras e espanholas com o diagnóstico de TDAH. O estudo é do tipo exploratório-descritivo, documental, retrospectivo, transversal, de caráter quantitativo dos prontuários de pacientes atendidos entre janeiro de 2010 a abril de 2015 no Hospital Universitário Virgen de la Arrixaca - Murcia/Espanha, e no Hospital Universitário Bettina Ferro de Souza - Belém/Brasil. Em comparação com as mães espanholas, as mães brasileiras relataram escores significativamente mais altos em quatro escalas, sendo elas: Internalização; Retraimento/Depressão; Queixas Somáticas e Problemas de Pensamento. Enquanto que, nas demais escalas, os escores foram relativamente similares em ambas as culturas. Estudos transculturais têm sido imprescindíveis para a melhor compreensão das semelhanças e diferenças das percepções de pessoas que vivem em culturas distintas.
\end{abstract}

Palavras-chave: Problemas de comportamento; Comparação transcultural; TDAH; CBCL.

\section{Perception of Brazilian and Spanish mothers about behavior problems in children with ADHD}

\begin{abstract}
Understanding the severity of the behavior problems can result in the development of the child throughout life, the aim of this study was to compare the perception of mothers in relation to behavior problems listed in the CBCL (Child Behavior Checklist) in two clinical samples composed of Brazilian and Spanish children diagnosed with ADHD. Method: The study is an exploratorydescriptive, documental, retrospective, cross-sectional, quantitative nature of the medical records treated between 2010 to April 2015 at the Hospital Universitario Virgen de la Arrixaca - Murcia/Spain, and the Hospital Universitário Bettina Ferro de Souza Belém/Brasil. Results: The Brazilian mothers reported significantly higher scores on four scales compared to the Spanish mothers, and in the other, the scores were relatively similar in both cultures. Conclusion: cross-cultural studies have been essential to a better understanding of the similarities and differences in perceptions of people living in different cultures.
\end{abstract}

Keywords: Behavior problems; Cross-cultural comparison; ADHD; CBCL.

\section{Percepción de madres brasileñas y españolas acerca de los problemas de conducta en hijos con TDAH}

\section{Resumen}

Comprendiendo la gravedad que los problemas de conducta pueden causar en el desarrollo del niño durante toda la vida, el objetivo de este estudio fue comparar la percepción de las madres en relación con los problemas de conducta que figuran en el CBCL (Child Behavior Checklist) en dos muestras clínicas compuestas de niños brasileños y españoles diagnosticados con TDAH. Método: El estudio es un documental-exploratorio descriptivo, transversal, de naturaleza cuantitativa retrospectiva de los informes de pacientes asistidos entre 2010 y abril de 2015 en el Hospital Universitario Virgen de la Arrixaca - Murcia/España, y en el Hospital Universitario de Bettina Ferro de Souza - Belém/Brasil. Resultados: Las madres brasileñas tuvieron puntuaciones significativamente mayores en cuatro escalas en comparación con las madres españolas, y en las demás, las puntuaciones fueron relativamente similares en ambas culturas. Conclusión: los estudios transculturales han sido esenciales para una mejor comprensión de las similitudes y diferencias en las percepciones de las personas que viven en las diferentes culturas.

Palabras clave: Problemas de conducta; Comparación intercultural; TDAH; CBCL. 
A cultura engloba padrões ou normas que influenciam o comportamento humano, como também, a maneira aceitável de se pensar. O que se desvia dessas concessões pode ser considerado anormal (Berry, Poortinga, Segal, \& Dasen, 2002; Nolen-Hoeksema, Fredrickson, Lofus, \& Wagenaar, 2012). A cultura funciona como uma catalizadora de significados, atuando na construção e organização mental da pessoa e, consequentemente, na formação social humana (Valsiner, 2012). Considerando a importância da compreensão do contexto cultural do indivíduo, estudos transculturais têm sido empregados por pesquisadores a fim de observar, testar e comparar participantes de diferentes culturas e circunferências geográficas em um ou mais aspectos do desenvolvimento humano (Egisdóttir, Gerstein, \& Çinarbas, 2008).

Estima-se que $13,4 \%$ da população mundial infantojuvenil é afetada por algum tipo de transtorno mental (Polanczyk, Salum, Sugaya, Caye, \& Rohde, 2015). Com base nisso, nas últimas décadas, o desenvolvimento socioemocional infantil tem despertado um crescente interesse na comunidade científica, especificamente, na identificação dos problemas comportamentais que podem predizer graves psicopatologias ao longo da vida (Dumas, 2011; Gómez, Santelices, Gómez, Rivera, \& Farkas, 2014; Whitbourne \& Halgin, 2015).

No relatório de saúde dos adolescentes ao redor do mundo elaborado pela Organização Mundial da Saúde (World Health Organization, 2014), a saúde mental é uma das prioridades da saúde pública. A OMS enfatiza que os transtornos mentais quando não são tratados podem acarretar em prejuízos significativos no desenvolvimento, como o suicídio, por exemplo, que ocupa o terceiro lugar no ranking de mortes na adolescência, e a depressão, que é um dos principais causadores de invalidez (World Health Organization, 2014).

Reconhece-se na literatura, que os problemas de comportamento envolvem desvios comportamentais que causam prejuízos ao indivíduo que o manifesta, como também, às pessoas que compartilham da sua convivência, ou seja, repercutem negativamente no âmbito intra e interpessoal (Bolsoni-Silva \& Del Prette, 2003). Em termos gerais, estes problemas são classificados em internalizantes e externalizantes (Archenbach \& Edelbrock, 1983).

Os problemas internalizantes abrangem os comportamentos de ordem privada, com sintomas relacionados à instabilidade de ânimo, ansiedade, depressão, isolamento social, retraimento, problemas somáticos, disforia, insegurança, timidez, entre outros (American Psychiatric Association, 2013; Archenbach \& Edelbrock, 1983; Hess \& Falcke, 2013; Rescorla et al., 2007). Já os externalizantes, são comportamentos dirigidos ao meio externo, evidenciados por oposição, condutas desafiadoras excessivas, manifestações antissociais, agressão física ou verbal, hostilidade, conduta disruptiva, impulsividade, uso de substâncias, entre outros sintomas (American Psychiatric Association, 2013; Archenbach \& Edelbrock, 1983; Rescorla et al., 2011).

Estudos sobre a prevalência dos problemas de comportamento em crianças e adolescentes apontam estimativas variadas. No Brasil, por exemplo, um estudo (Borsa \& Nunes, 2011a) com 366 escolares de 6 a 12 anos, utilizando o instrumento CBCL, estimou a porcentagem de $39,6 \%$ de comportamentos internalizantes e 30,6\% de externalizantes. Por sua vez, uma investigação na Espanha (Fernández-Molina, Valle, Fuentes, Isabel María Bernedo, \& Bravo, 2011) com 181 adolescentes de 11 a 18 anos, também utilizando o CBCL como instrumento de coleta, encontrou a taxa de $24,3 \%$ de comportamentos internalizantes e $28,7 \%$ de externalizantes.

Dentre os transtornos do neurodesenvolvimento, o Transtorno de Déficit de Atenção e Hiperatividade (TDAH), é um transtorno que possui considerável incidência comórbida com os problemas de comportamento. A exemplo disso, um estudo realizado em Porto Rico (Rivera, Martínez, \& Pérez, 2005) comparou a sintomatologia internalizante e externalizante em 90 estudantes na faixa etária de 8 a 12 anos, sendo que, 45 possuíam o diagnóstico de TDAH, e 45 do grupo controle sem o transtorno. Os pesquisadores apontaram que a média de comportamentos internalizantes e externalizantes, tais como, depressão, ansiedade, irritabilidade e hostilidade, foi superior na amostra com TDAH em comparação ao grupo controle, variando de 2,02 a 7,01 pontos de diferença. Outros investigadores também encontraram relação entre tais sintomas e o TDAH (Jacob et al., 2014; Kuja-Halkola, Lichtenstein, D'Onofrio, \& Larsson, 2015; López Soler et al., 2009; Navarro \& García-Villamisar, 2011; Treuting \& Hinshaw, 2001).

O TDAH inicia-se na infância e tem como marco diagnóstico a tríade sintomatológica de déficit de atenção, hiperatividade e impulsividade, que se manifestam de maneira desproporcional em relação à idade biológica e ao nível de desenvolvimento do indivíduo (American Psychiatric Association, 2013; Barkley, 2008; Cunill \& Xavier, 2015). O padrão inconsistente da desatenção, por exemplo, caracteriza-se pela dificuldade em sustentar a atenção nas tarefas, distraibilidade, divagação, falta de persistência e desorganização (American Psychiatric Association, 2013). Enquanto que, a hiperatividade, evidencia-se 
por instabilidade e inquietação motora excessiva, interferindo na socialização do indivíduo (VaquerizoMadrid, 2005). Por sua vez, a impulsividade refere-se à dificuldade em postergar as gratificações, retardar respostas, planejar, déficit na regulação inibitória do comportamento, caracterizando um fragilizado funcionamento executivo (Barkley, 2008).

Em relação ao TDAH e os problemas de comportamento, são escassos na literatura os estudos transculturais, principalmente, comparando o Brasil e a Espanha, dois países com histórias e culturas distintas. Estudos transculturais têm sido imprescindíveis para a melhor compreensão das diferenças perceptuais das pessoas que vivenciam culturas distintas, e, consequentemente, viabiliza a análise dos possíveis efeitos da mesma na modelagem, interpretação e expressão dos comportamentos dos indivíduos no contexto familiar e nos demais sistemas sociais (American Psychiatric Association, 2013; Olatundun, 2009; Whitbourne \& Halgin, 2015).

A cultura repercute na valoração do comportamento esperado e tolerável da criança em relação ao seu período do desenvolvimento, tanto na percepção dos pais, como na avaliação e classificação dos comportamentos psicopatológicos por profissionais (American Psychiatric Association, 2013; Bauermeister, 2014; Brewis, Schmidt, \& Casas, 2003; Whitbourne \& Halgin, 2015). Neste processo, instrumentos de avaliação comportamental têm sido ferramentas essenciais na investigação das diferenças individuais (Bechtold, Bhawuk, \& Lee, 2002; Rohde, 2002).

Dos instrumentos de análise comportamental, o CBCL (Child Behavior Checklist) tem se destacado entre os profissionais no rastreamento dos problemas de comportamento de crianças e adolescentes. Este instrumento está integrado ao Sistema de Avaliação de Base Empírica de Archenbach - ASEBA (Archenbach \& Edelbrock, 1983). O CBCL, especificamente, avalia a percepção dos pais sobre o comportamento dos filhos. Sua validade multicultural já foi confirmada em várias nacionalidades.

Utilizando o CBCL, Rescorla et al., (2007) comparou 31 sociedades diferentes (Austrália, Bélgica, China, Dinamarca, Etiópia, Finlândia, França, Alemanha, Grécia, Hong Kong, Irã, Israel, Itália, Islândia, Jamaica, Japão, Coreia, Lituânia, Holanda, Noruega, Polônia, Portugal, Porto Rico, Romênia, Rússia, Suécia, Suíça, Taiwan, Tailândia, Turquia, e Estados Unidos), investigando a avaliação dos pais em relação aos problemas de comportamento dos filhos de 6 a 16 anos, e constataram similaridades dos escores encontrados nas diferentes culturas. Em todos estes países, foram avaliados os efeitos do país, gênero e faixa etária nos comportamentos dos testados (Rescorla et al., 2007).

Compreendendo o papel da cultura, a gravidade que os problemas de comportamento podem acarretar no desenvolvimento da criança ao longo da vida, e a originalidade do presente estudo ao comparar o Brasil e a Espanha, não foram delimitadas hipóteses específicas. Em vez disso, foram desenvolvidas três questões gerais para nortear a discussão, sendo elas: (1) A cultura influenciará na percepção das mães na observação do comportamento dos filhos? (2) As variáveis gênero e faixa etária influenciarão nos escores das escalas? (3) Haverá diferença significativa nas escalas de externalização e internalização? Sendo assim, o objetivo do presente estudo foi comparar os problemas de comportamentos em duas amostras clínicas de crianças brasileiras e espanholas com TDAH.

\section{Método}

\section{Delineamento}

O estudo é do tipo exploratório-descritivo, comparativo, documental, retrospectivo, transversal, de caráter quantitativo. A pesquisa documental foi realizada por meio da análise dos prontuários de pacientes diagnosticados com TDAH em duas unidades distintas de psicologia de hospitais universitários, voltadas para o público infanto-juvenil.

\section{Campo de estudo}

Uma parte da pesquisa foi realizada na Unidade Clínica da Infância e Adolescência do Hospital Universitário Virgen de la Arrixaca, vinculado à Universidad de Murcia, Espanha. E a segunda parte ocorreu na Unidade de Psicologia do Serviço de Crescimento e Desenvolvimento CAMINHAR, voltada para o atendimento infantil, do Hospital Universitário Bettina Ferro de Souza, vinculado à Universidade Federal do Pará, localizado na cidade de Belém do Pará, Brasil.

\section{Participantes}

Foi feita a análise documental retrospectiva de todos os prontuários atendidos entre janeiro de 2010 a abril de 2015 na unidade hospitalar de Murcia e na unidade do Bettina. Esta fase inicial consistiu na análise de 1.530 prontuários na Espanha e 1.645 no Brasil. Destes, foram selecionados 67 prontuários na unidade hospitalar de Murcia e 94 na unidade do Bettina, uma vez que estes atenderam aos critérios de inclusão estabelecidos, isto é, ter o diagnóstico de TDAH e ter respondido ao $\mathrm{CBCL} / 6-18$ anos como 
parte do processo diagnóstico. Estes critérios foram escolhidos com a finalidade de uniformizar a amostra nos dois países uma vez que a unidade na Espanha é voltada para o público infanto-juvenil, enquanto que, no Brasil, o público é infantil. Assim, foram excluídos os prontuários que não registravam o diagnóstico de TDAH; prontuários de pacientes na faixa etária entre 13 a 18 anos, prontuários com o CBCL/1(1/2)-5 anos utilizados para crianças com a idade entre 1 a 5 anos e prontuários de pacientes que apresentavam apenas a sintomatologia do transtorno, mas não atendiam a todos os critérios diagnósticos delimitados no DSMIV e CID-10, sistemas de classificação utilizados nas duas unidades.

O total de participantes foi de $n=105$ prontuários cujas informações registradas foram obtidas mediante o relato de mães de crianças na faixa etária de 6 a 12 anos. A amostra brasileira totalizou em 70 prontuários, sendo 18 de crianças do sexo feminino, e 52 do sexo masculino; enquanto que, a amostra espanhola consistiu em 35 prontuários, sendo 7 de crianças do sexo feminino e 28 do sexo masculino.

\section{Instrumento}

O CBCL/6-8 é composto de uma lista de 113 itens que avaliam os problemas emocionais/ comportamentos e habilidades sociais (Achenbach \& Rescorla, 2001). Os 113 itens geram escores em oito síndromes que avaliam dimensões psicopatológicas: Ansiedade/Depressão; Retraimento/Depressão; Queixas somáticas; Problemas de sociabilidade; Problemas de pensamento; Problemas de atenção; Violação de regras; e Comportamento agressivo; sendo que as três primeiras síndromes (Ansiedade/ Depressão; Retraimento/Depressão; Queixas somáticas) compõem uma escala maior denominada de Escala de Internalização, as duas últimas (violação de regras e comportamento agressivo) integram a Escala de Externalização, e as duas escalas (problemas de pensamento e problemas de atenção) compõem a escala maior intitulada de Outros Problemas (Achenbach \& Rescorla, 2001). As respostas dos itens variam entre 0 (zero) que corresponde a comportamento ausente; 1 (um) comportamento às vezes presente e; 2 (dois) comportamento frequentemente presente.

\section{Implicações éticas}

Para a realização do estudo, foi assegurado aos participantes o caráter confidencial e sigiloso dos pacientes sem oferecer qualquer tipo de risco. Foi solicitada autorização do Comitê de Ética do Hospital Virgen de la Arrixaca (CEIC Virgen de la Arrixaca), que emitiu parecer favorável (DICTAMEN FAVORABLE), na Ata 08/15 para a coleta de dados. Procedimento semelhante foi adotado no contexto brasileiro, onde o projeto foi submetido ao Comitê de Ética do Núcleo em Medicina Tropical da UFPA que autorizou sua realização no Hospital Bettina Ferro de Souza em 10/2015.

\section{Análise dos dados}

Para a análise estatística dos resultados encontrados foram aplicadas as técnicas estatísticas, descritiva e inferencial. Na descritiva foram construídas tabelas contendo valores absolutos e relativos para facilitar na descrição dos resultados confirmados por meio da análise inferencial.

Para analisar inferencialmente os resultados organizados na análise descritiva, foram aplicados os testes Kolmogorov-Smirnov, e o de Levene para testar os pressupostos de Normalidade e Igualdade de Variâncias para aplicação dos testes paramétricos de comparação de médias, $t$ de Student e Análise de Varância (ANOVA). Também foram aplicados os testes não-paramétricos, Qui-quadrado de aderência para proporções esperadas iguais e Qui-quadrado de Independência com objetivo de investigar a existência de relação estatisticamente significante entre as variáveis e os grupos de interesse na pesquisa, considerando o nível de significância $\alpha=0,05$.

Utilizou-se o programa software ADM (Assessment Data Manager) criado por Achenbach e colaboradores (Achenbach System of Empirically Based Assessment, 2006) para a análise dos perfis. Os programas utilizados para construção do banco de dados e aplicação dos testes estatísticos foram o SPSS 22.0 (StatisticalPackage for Social Science) na versão 22.0.

\section{Resultados}

$\mathrm{Na}$ análise total dos problemas de comportamento avaliados pelo CBCL, consideraram-se satisfatório o teste $t$, ANOVA e os pressupostos de Normalidade e Igualdade de Variâncias (Homocedasticidade) por país, gênero e faixa etária (6-7anos, 8-9 anos, 10-12 anos). Na maioria das escalas, as crianças brasileiras alcançaram escore superior ao das crianças espanholas, mostrando que a percepção das mães revela um perfil cultural relativamente estável.

Análise do teste $t$ para amostras independentes foi conduzida na comparação das respostas do CBCL das mães das duas nacionalidades, Brasil $(\mathrm{N}=70)$ e Espanha $(\mathrm{N}=35)$. Apresenta-se na Tabela 1, os resultados dos testes utilizados para verificar as diferenças nos escores obtidos com país de crianças com TDAH nas escalas de internalização e externalização. 
TABELA 1

Escores das síndromes do CBCL

\begin{tabular}{|c|c|c|c|c|c|}
\hline \multirow[t]{2}{*}{ Sindromes } & $\begin{array}{c}\text { Brasil } \\
(N=70)\end{array}$ & $\begin{array}{c}\text { Espanha } \\
(N=35)\end{array}$ & \multirow[t]{2}{*}{ Diferença de médias } & \multirow[t]{2}{*}{$t$} & \multirow[t]{2}{*}{ p-valor } \\
\hline & Média $(D P)$ & Média $(D P)$ & & & \\
\hline Escala de Internalização & $19,49(9,15)$ & $15,06(8,13)$ & 4,42 & 2,42 & $0,017 *$ \\
\hline Escala de Externalização & $21,01(11,41)$ & $18,71(9,60)$ & 2,30 & 1,02 & 0,308 \\
\hline Outros Problemas & $8,74(4,38)$ & $7,29(3,29)$ & 1,45 & 1,73 & 0,086 \\
\hline Ansiedade/Depressão & $8,63(4,37)$ & $7,86(4,79)$ & 0,77 & 0,82 & 0,411 \\
\hline Retraimento/Depressão & $5,24(3,27)$ & $3,40(2,73)$ & 1,84 & 2,86 & $0,005^{*}$ \\
\hline Queixas Somáticas & $5,61(4,06)$ & $3,80(3,01)$ & 1,81 & 2,33 & $0,021 *$ \\
\hline Problemas de Sociabilidade & $8,87(4,78)$ & $7,74(4,27)$ & 1,12 & 1,17 & 0,241 \\
\hline Problemas de pensamento & $8,44(5,67)$ & $5,60(5,15)$ & 2,84 & 2,49 & $0,014 *$ \\
\hline Problemas de Atenção & $11,29(4,82)$ & $12,63(4,15)$ & $-1,34$ & $-1,40$ & 0,163 \\
\hline Violação de Regras & $5,76(4,79)$ & $5,17(3,66)$ & 0,59 & 0,63 & 0,527 \\
\hline Comportamento Agressivo & $15,26(8,02)$ & $13,54(6,72)$ & 1,72 & 1,08 & 0,280 \\
\hline
\end{tabular}

* $p<0,05$ (Teste $t$ de Student).

Em termos gerais, observa-se na Tabela 1 que as médias encontradas nas Escalas de Internalização e Outros Problemas foram inferiores nos dois países quando se compara a Escala de Externalização, sugerindo alta frequência percepção das mães de crianças com TDAH de comportamentos externalizantes.

No que tange ao país, encontrou-se resultado significativo na Escala de Internalização (ver Tabela 1) em que se confirma os escores superiores das brasileiras $(\mathrm{M}=19,49, \mathrm{DP}=9,15)$ com relação as espanholas $(\mathrm{M}=15,06, \mathrm{DP}=8,13), t=2,42, p<0,05$. Os resultados indicam maior tendência das mães brasileiras na percepção dos comportamentos de ordem privada que as espanholas.

Por sua vez, na Escala de Externalização (ver Tabela 1), a diferença de médias de 2,30 foi insignificante, sugerindo que as mães brasileiras e espanholas percebem com uma certa semelhança os comportamentos externalizantes nos filhos com TDAH. Quanto a Escala de Outros Problemas, também não foi encontrada diferença significativa entre os dois países, indicando que as participantes se aproximam em termos de percepção dos outros problemas nos filhos.

Conforme se observa na Tabela 1, foram encontrados resultados significativos em três síndromes. Especificamente, nos problemas de comportamento que integram a Escala de Internalização, as mães brasileiras reportaram escores significativamente superiores na síndrome de Retraimento/Depressão $(M=5,24$; $\mathrm{DP}=3,27)$, que as espanholas $(\mathrm{M}=3,40 ; \mathrm{DP}=2,73)$, $t=2,8, p<0,05$; como também, na síndrome de Queixas
Somáticas $(\mathrm{M}=5,61, \mathrm{DP}=4,06)$, em comparação à média das espanholas $(\mathrm{M}=3,80, \mathrm{DP}=3,01), t=2,3$; $p<0,05$.

No que tange à competência social avaliada por meio da escala de Problemas de Sociabilidade, não foi encontrada significância na comparação dos dois países, tampouco na síndrome de Problemas de Atenção, dado que aponta a acurácia do CBCL como um instrumento complementar na detecção do déficit de atenção, já que a amostra é composta por crianças diagnosticadas com TDAH (ver Tabela 1), indicando que as mães percebem a ocorrência do comportamento com frequência similar.

Em relação aos Problemas de Pensamento, o escore das mães brasileiras foi significativamente superior ao das espanholas, sinalizando que as brasileiras observam com mais frequência nos filhos os sintomas de problemas de pensamento. Em contrapartida, nas síndromes de Violação de Regras e Comportamento Agressivo que integram a Escala de Externalização, não foi encontrada diferença significativa nas médias, o que sugere que tais comportamentos são percebidos de modo semelhante nos dois países pelas mães, nos filhos com TDAH.

A análise de variância (ANOVA) foi aplicada às variáveis gênero e idade de cada país, no entanto, não foram encontradas diferenças significativas. $\mathrm{Na}$ variável gênero, observou-se que, embora a amostra feminina fosse inferior à masculina em ambos os países, encontraram-se médias superiores na percepção das mães de meninas em algumas síndromes em relação à percepção das mães de meninos (ver Tabela 2). 
TABELA 2

Escore do CBCL em função do gênero

\begin{tabular}{|c|c|c|c|c|c|c|}
\hline \multirow{3}{*}{ Sindromes/Escalas } & \multicolumn{2}{|c|}{$\begin{array}{c}\text { Brasil } \\
(N=70)\end{array}$} & \multicolumn{2}{|c|}{$\begin{array}{c}\text { Espanha } \\
(N=35)\end{array}$} & \multirow{3}{*}{$F$} & \multirow{3}{*}{$p$} \\
\hline & $\begin{array}{l}\text { Menino } \\
(N=52)\end{array}$ & $\begin{array}{l}\text { Menina } \\
(N=18)\end{array}$ & $\begin{array}{l}\text { Menino } \\
(N=28)\end{array}$ & $\begin{array}{c}\text { Menina } \\
(N=7)\end{array}$ & & \\
\hline & Média $(D P)$ & Média $(D P)$ & Média $(D P)$ & Média $(D P)$ & & \\
\hline Ansiedade/Depressão & $8,44(4,14)$ & $9,17(5,06)$ & $8,04(4,79)$ & $7,14(5,11)$ & 0,010 & 0,941 \\
\hline Retraimento/Depressão & $5,56(3,39)$ & $4,33(2,78)$ & $3,57(2,82)$ & $2,71(2,43)$ & 1,778 & 0,185 \\
\hline Queixas Somáticas & $5,23(3,77)$ & $6,72(4,75)$ & $3,46(2,86)$ & $5,14(3,48)$ & 2,856 & 0,094 \\
\hline Problemas de Sociabilidade & $9,27(4,71)$ & $7,72(4,93)$ & $7,75(4,61)$ & $7,71(2,75)$ & 0,460 & 0,498 \\
\hline Problemas de Pensamento & $8,71(5,53)$ & $7,67(6,16)$ & $5,25(3,83)$ & $7,00(9,03)$ & 0,064 & 0,800 \\
\hline Problemas de Atenção & $11,54(4,80)$ & $10,56(4,94)$ & $12,96(3,75)$ & $11,29(5,64)$ & 1,305 & 0,256 \\
\hline Violação de Regras & $5,85(5,11)$ & $5,50(3,854)$ & $5,43(3,67)$ & $4,14(3,71)$ & 0,52 & 0,471 \\
\hline Comportamento Agressivo & $15,08(7,99)$ & $15,78(8,32)$ & $13,89(7,05)$ & $12,14(5,39)$ & 0,073 & 0,786 \\
\hline Escala de Internalização & $20,63(11,04)$ & $20,78(11,21)$ & $19,43(10,03)$ & $14,86(8,13)$ & 0,681 & 0,411 \\
\hline Escala de Externalização & $20,11(9,66)$ & $19,67(9,38)$ & $15,00(8,67)$ & $15,04(8,15)$ & 0,007 & 0,930 \\
\hline
\end{tabular}

$\mathrm{p}<0,0001$.

No Brasil, por exemplo, a percepção das mães de meninas, foi superior nas síndromes de Ansiedade/ Depressão e Queixas Somáticas, enquanto que, na Espanha, as meninas apresentaram médias superiores à dos meninos nas síndromes de Queixas Somáticas e Problemas de Pensamento. Nas demais síndromes, os meninos obtiveram escores mais elevados. Todavia, ressalta-se que não foram encontradas diferenças significativas em função do sexo em nenhuma síndrome (ver Tabela 2).

No geral, as mães de meninos brasileiros, apresentaram escores mais elevados que a das mães de meninos espanhóis (ver Tabela 2), exceto na síndrome de Problemas de Atenção, pois no grupo da Espanha foi encontrado média superior. Por sua vez, ao se comparar as meninas brasileiras e espanholas, verificase que as brasileiras se sobressaíram na maioria das síndromes, exceto na de Problemas de Atenção, em que as espanholas apresentaram média superior. Em relação a Escala de Internalização e Externalização, os escores das mães brasileiras foram superiores em ambos os sexos, uma vez comparadas as médias das mães espanholas. Todavia, enfatiza-se que não foram encontradas diferenças significativas.

Em relação à variável idade (ver Tabela 3), foram comparados três grupos de faixa etária por país (6-7anos; 8-9 anos; e 10-12 anos). No entanto, não foram encontradas diferenças significativas tanto nas síndromes quanto nas escalas. As médias variaram em conformidade com a síndrome avaliada, não havendo, portanto, uma uniformidade de grupo que se superou integralmente ao outro, em ambos os países (ver Tabela 3). No Brasil, as idades que alcançaram as maiores médias foram a de 6 e 7 anos, enquanto que, na Espanha, foi o grupo de 8 a 9 anos.

$\mathrm{Na}$ Escala de Internalização, por exemplo, no Brasil, as crianças de 6 a 7 anos apresentaram um escore superior em relação aos demais grupos etários $(\mathrm{M}=24,73 ; \mathrm{DP}=11,10)$. Por outro lado, na Espanha nessa mesma Escala, a maior média foi encontrada no grupo de faixa etária de 10 a 12 anos $(\mathrm{M}=19,83$; $\mathrm{DP}=14,41)$. Já na Escala de Externalização, no Brasil, o maior escore foi na idade de 6 a 7 anos $(M=20,47$; $\mathrm{DP}=11,90)$; e na Espanha, foi no grupo de 8 a 9 anos $(\mathrm{M}=16,88 ; \mathrm{DP}=8,58)$.

Nas síndromes que integram a Escala de Internalização (Ansiedade/Depressão; Retraimento/Depressão; e Queixas Somáticas), no Brasil, respectivamente, os maiores escores foram nos grupos de 6 a 7 anos $(\mathrm{M}=9,40 ; \mathrm{DP}=4,50) ; 10$ a 12 anos $(\mathrm{M}=5,48 ; \mathrm{DP}=3,43)$; e 6 a 7 anos $(M=5,93 ; D P=5,351)$. Ao passo que, na Espanha, as médias mais altas foram nos grupos de 10 a 12 anos $(M=7,83 ; \mathrm{DP}=6,49) ; 8$ a 9 anos $(\mathrm{M}=4,19$; $\mathrm{DP}=2,90)$; e 8 a 9 anos $(\mathrm{M}=4,19$; $\mathrm{DP}=2,56)$.

Nas síndromes que integram a Escala de Externalização (Violação de Regras e Comportamento Agressivo), respectivamente, no Brasil, os maiores escores foram nos grupos de 6 a 7 anos em ambas as síndromes $(\mathrm{M}=6,64 ; \mathrm{DP}=4,44 / \mathrm{M}=18,43 ; \mathrm{DP}=8,53)$. Por outro lado, na Espanha, as médias variaram, na Violação de Regras, por exemplo, o escore foi maior nas idades de 8 a 9 anos $(M=5,62$; $\mathrm{DP}=5,12)$, e na síndrome de Comportamento Agressivo, destacou-se o grupo de 10 a 12 anos $(\mathrm{M}=14,75 ; \mathrm{DP}=4,30)$. 
TABELA 3

Escore do CBCL em função da faixa etária

\begin{tabular}{|c|c|c|c|c|c|c|c|c|}
\hline \multirow{3}{*}{ Sindromes /Escalas } & \multicolumn{3}{|c|}{$\begin{array}{c}\text { Brasil } \\
(N=70)\end{array}$} & \multicolumn{3}{|c|}{$\begin{array}{c}\text { Espanha } \\
(N=35)\end{array}$} & \multirow{3}{*}{$F$} & \multirow{3}{*}{$p$} \\
\hline & 6 a 7 anos & 8 a 9 anos & 10 a 12 anos & 6 a 7 anos & 8 a 9 anos & 10 a 12 anos & & \\
\hline & Média $(D P)$ & Média $(D P)$ & Média $(D P)$ & Média (DP) & Média $(D P)$ & Média $(D P)$ & & \\
\hline Ansiedade/Depressão & $9,40(4,50)$ & $7,57(3,92)$ & $9,30(4,67)$ & $7,00(4,22)$ & $8,56(4,76)$ & $7,83(6,49)$ & 0,0803 & 0,9229 \\
\hline Retraimento/Depressão & $5,33(4,11)$ & $4,96(2,67)$ & $5,48(3,43)$ & $2,85(2,57)$ & $4,19(2,90)$ & $2,50(2,42)$ & 0,3157 & 0,7300 \\
\hline Queixas Somáticas & $5,93(5,35)$ & $5,68(3,49)$ & $5,37(3,94)$ & $3,38(3,99)$ & $4,19(2,56)$ & $3,67(1,75)$ & 0,0909 & 0,9131 \\
\hline Problemas de Sociabilidade & $9,07(5,87)$ & $7,75(4,52)$ & $9,93(4,28)$ & $8,23(4,71)$ & $8,00(4,17)$ & $6,00(3,74)$ & 0,2504 & 0,7790 \\
\hline Problemas de Pensamento & $9,07(5,87)$ & $7,75(4,52)$ & $9,93(4,28)$ & $8,23(4,71)$ & $8,00(4,17)$ & $6,00(3,74)$ & 0,7438 & 0,4779 \\
\hline Problemas de Atenção & $12,27(4,18)$ & $10,68(4,78)$ & $11,37(5,24)$ & $13,92(3,77)$ & $13,19(3,77)$ & $11,29(5,64)$ & 1,305 & 0,2560 \\
\hline Violação de Regras & $6,64(4,44)$ & $5,23(5,59)$ & $5,88(4,01)$ & $4,20(2,74)$ & $5,62(5,12)$ & $5,50(2,35)$ & 0,0377 & 0,9630 \\
\hline Comportamento Agressivo & $18,43(8,53)$ & $12,93(6,76)$ & $16,23(8,58)$ & $13,30(8,28)$ & $12,62(7,55)$ & $14,75(4,30)$ & 1,6165 & 0,2038 \\
\hline Escala de Internalização & $24,73(11,10)$ & $17,18(9,98)$ & $22,04(11,26)$ & $19,23(8,73)$ & $17,44(9,09)$ & $19,83(14,41)$ & 0,8465 & 0,1632 \\
\hline Escala de Externalização & $20,47(11,90)$ & $19,07(8,05)$ & $20,15(9,45)$ & $15,23(7,96)$ & $16,88(8,58)$ & $14,00(7,48)$ & 0,1442 & 0,8659 \\
\hline
\end{tabular}

$p<0,0001$.

Na síndrome de Problemas de Sociabilidade, no Brasil, a média que se destacou foi a do grupo de 10 a 12 anos $(M=9,93 ; D P=4,28)$. Já na Espanha, foram as idades de 6 a 7 anos $(\mathrm{M}=8,23$; $\mathrm{DP}=4,711)$. Por sua vez na síndrome de Problemas de Pensamento, no Brasil, destacou-se o grupo de 10 a 12 anos $(M=9,93$; $\mathrm{DP}=4,28)$; e na Espanha as idades de 6 a 7 anos $(\mathrm{M}=8,23 ; \mathrm{DP}=4,71)$.

Por fim, na síndrome de Problemas de Atenção, no Brasil, o grupo de 6 a 7 anos apresentou o maior escore $(M=12,27 ; \mathrm{DP}=4,18)$; e, coincidentemente, na Espanha, o mesmo grupo apresentou a maior média $(\mathrm{M}=13,92 ; \mathrm{DP}=3,77)$, sugerindo que as mães das duas nacionalidades, observam com mais frequência os problemas atencionais nesta faixa etária.

\section{Discussão}

Buscou-se na literatura científica, estudos que objetivassem comparar a percepção das mães brasileiras e espanholas, no entanto, não foram encontrados artigos com tal perspectiva, dado que aponta a sua originalidade. O presente estudo ao equiparar as duas amostras clínicas independentes por país, gênero e faixa etária, possibilitou a análise da frequência de problemas de comportamentos das crianças com TDAH, a fim de evidenciar os principais sintomas presentes nas duas culturas.

Os resultados sinalizaram escores relativamente similares nos dois países. As mães brasileiras e espanholas evidenciaram maiores médias na Escala de Externalização, seguidas das síndromes de Com- portamentos Agressivo e Problemas de Atenção. Estes achados corroboram vários registros na literatura científica que apontam um perfil psicopatológico comórbido do TDAH com os comportamentos externalizantes (Jacob et al., 2014; Kuja-Halkola et al., 2015; Rivera et al., 2005; Roessner, Becker, Rothenberger, Rohde, \& Banaschewski, 2007; Rohde et al., 2005; Schoemaker et al., 2013; Tureck, Matson, May, \& Turygin, 2013). Este achado converge com o estudo de comparação transcultural realizado entre Brasil e Alemanha (Roessner et al., 2007), que sugeriu ser um padrão consistente que independe da cultura.

A associação do TDAH com problemas externalizantes pode ajudar a entender as evidências apontadas nas pesquisas que relacionam este transtorno à vulnerabilidade destas crianças e adolescentes que estão mais suscetíveis à reprovação, expulsão e abandono escolar, ao abuso de drogas lícitas e ilícitas, às práticas de riscos, delituosas e antissociais, à violação de regras, aos comportamentos agressivos (Barbaresi et al., 2013; Bauermeister, 2014; López-Soler \& Romero Medina, 2013; Miranda, Colomer, Berenguer, Roselló, \& Roselló, 2015; Ohlmeier et al., 2008; Possa, Spanemberg, \& Guardiola, 2005). Os problemas de conduta observados geralmente resultam em desafios para os pais, familiares e cuidadores, pois dificultam na aprendizagem de comportamentos adaptativos positivos, no autocontrole, na autorregulação das emoções e na aquisição de habilidades sociais necessárias nas relações interpessoais (Tureck et al., 2013).

Em relação à síndrome de Problemas de Atenção, nos dois países foram encontrados altos escores, 
sinalizando acurácia do CBCL como um instrumento de triagem no processo diagnóstico do TDAH. Tal tendência já foi verificada em outras investigações que assinalaram a acurácia do instrumento (Lampert, 2002; Roessner et al., 2007).

$\mathrm{Na}$ análise da variável país, as mães brasileiras apresentaram escores superiores nas síndromes e escalas quando comparadas às espanholas, exceto na média da síndrome de Problemas de Atenção. Todavia, encontraram-se escores significativamente diferentes somente na Escala de Internalização, e nas síndromes de Retraimento/Depressão, Queixas Somáticas, e Problemas de Pensamento. Este resultado possivelmente está relacionado à superestimação de doenças nos filhos por parte das brasileiras, à desigualdade socioeconômica (Rivera et al., 2005; Roessner et al., 2007), e à influência da cultura na interpretação das perguntas do CBCL (Rescorla et al., 2007; Tsiantis, Motti-Stefanidi, Richardson, Schmeck, \& Poustka, 1994). Alguns estudos brasileiros assinalaram alta prevalência de comportamentos internalizantes em escolares, sugerindo uma tendência das brasileiras em perceber os comportamentos de ordem privada (Borsa \& Nunes, 2011b; Borsa, Souza, \& Bandeira, 2011).

Em relação à variável gênero, não foram encontradas diferenças significativas ao comparar meninas e meninos em ambas as nacionalidades. Não há um consenso na literatura sobre este achado, inclusive são limitadas as investigações utilizando o CBCL com amostra similar ao presente estudo. A despeito das controvérsias em torno deste tema, alguns autores sinalizam maior prevalência dos comportamentos internalizantes nas meninas e dos comportamentos externalizantes nos meninos (LópezSoler, Sáez, López, Fernández, \& Pina, 2009; Rivera et al., 2005; Skogli, Teicher, Andersen, Hovik, \& Øie, 2013). Estudos realizados com amostra de escolares sem transtornos psicológicos apontaram resultados divergentes (Borsa \& Nunes, 2011b; Borsa et al., 2011; Emerich, Da Rocha, Silvares, \& Gonçalves, 2012).

No que tange à variável idade, os achados não apontaram diferenças significativas. Utilizando o CBCL, um estudo multicultural de alta relevância realizado em 31 países situados nos continentes Americano, Asiático, Europeu e Oceania (Rescorla et al., 2011) sinalizou inconsistência nas sociedades analisadas cujos resultados variaram em função da idade, como, por exemplo, na maioria dos países, o gênero feminino de 12 a 16 anos, apresentou escores mais elevados nos comportamentos internalizantes, ou seja, as meninas de 6 a 11 anos não apontaram diferença significativa. Já os meninos 6 a 11 anos alcançaram escores mais elevados nos comportamentos externalizantes, mas isso não se aplicou em todas as sociedades.

A partir da exposição dos achados, convém discutir alguns fatores que possivelmente influenciaram nos resultados. Na avaliação comportamental de crianças, por exemplo, se faz necessária a participação dos pais ou cuidadores nas informações pertinentes ao contexto e ao comportamento, uma vez que estes participam diretamente do processo de desenvolvimento e maturação psicológica da criança (Whitbourne \& Halgin, 2015). Todavia, estudos (Bauermeister, 2014; Foley et al., 2005) apontam que diversas variáveis podem influenciar na forma como os pais percebem os comportamentos dos seus filhos, tais como o histórico de psicopatologias, conflitos familiares, e a cultura.

A exemplo disso, um estudo realizado no Brasil (Borsa \& Nunes, 2008), objetivou averiguar a concordância entre as respostas das mães e pais aos problemas comportamentais avaliados pelo CBCL, e os resultados apontaram baixa concordância entre as respostas, sinalizando como possível influência os aspectos sociais que delimitam os papéis dos genitores na família e os conflitos na relação intrafamiliar. Ressalta-se que os autores sugerem que em comparação aos pais, as mães tendem a ser mais precisas na avaliação do comportamento dos filhos.

Na Espanha, pesquisadores (Pérez-López, CanoGiménez, \& Agustín, 2009), compararam a percepção dos genitores e dos educadores de escola infantil acerca dos problemas comportamentais utilizando o CBCL como um dos instrumentos, e constataram que as mães percebem mais claramente os problemas comportamentais dos filhos que os seus educadores escolares, resultado também encontrado em outro estudo chileno (Gómez et al., 2014).

As informações prestadas pelos pais respondentes dos testes também podem estar relacionadas à cultura de cada país, aos aspectos sociais, a subjetividade de cada respondente na interpretação das perguntas, aos conflitos familiares, entre outros fatores (Barkley, 2008; Bauermeister, 2014; Pires et al., 2012; Schroeder \& Kelley, 2009).

\section{Considerações Finais}

A principal contribuição deste estudo foi identificar as diferenças e características comportamentais similares em crianças alocadas em países distintos, por meio das respostas das mães ao instrumento CBCL. Todavia, algumas limitações necessitam ser observadas. Primeiro, as amostras utilizadas em ambos os países não representam a população geral brasileira e espanhola. Segundo, realizar estudos transculturais 
demanda tempo, disponibilidade de outros profissionais, enfrentamento de burocracias internas e éticas nas unidades de pesquisa, e diferenças de fusos horários, variáveis que possivelmente influenciaram o $\mathrm{N}$ da amostra. Além disso, houve a ausência de grupo controle.

Um comparativo do CBCL com outras escalas que avaliam o funcionamento executivo, com certeza, repercutiria em dados mais detalhados e precisos sobre os prejuízos no desenvolvimento funcional dessas crianças, todavia, a falta de validação de outras medidas no contexto brasileiro inviabilizou tal análise.

O TDAH é uma realidade atestada por diversos estudos de prevalência realizados países diferentes, assim como as suas comorbidades. Então, entende-se que estudos transculturais podem contribuir na medida em que permitem identificar semelhanças e diferenças, especificidades e generalidades o que pode favorecer a construção de um conhecimento que ajude a explicar o TDAH e, consequentemente, suas implicações na vida das pessoas.

\section{Referências}

Achenbach System of Empirically Based Assessment. (2006). Manual for the assessment data manager program $(A D M)$ (VT). Burlington.

American Psychiatric Association. (2013). Diagnostic and statistical manual of mental disorders (4th ed., text rev.). Washington: Am. Psychiatr. Publ. https://doi.org/10.1176/appi.books.9780890425596

Archenbach, T. \& Edelbrock, C. (1983). Manual of child behavior checklist and revised child behavior profile. Burlington: University of Burlington/Departament of Psychiatric.

Barbaresi, W. J., Colligan, R. C., Weaver, A. L., Voigt, R. G., Killian, J. M., \& Katusic, S. K. (2013). Mortality, ADHD, and psychosocial adversity in adults with childhood ADHD: a prospective study. Pediatrics, 131(4), 637-644. https:// doi.org/10.1542/peds.2012-2354

Barkley, R. A. (2008). Transtorno do Déficit de Atenção/Hiperatividade: Manual para diagnóstico e tratamento. Porto Alegre: Artmed.

Bauermeister, J. J. (2014). Hiperactivo, impulsivo, distraido ¿Me conoces? (3ª ed.). New York: The Guilford Press.

Bechtold, D. J., Bhawuk, D. P. S., \& Lee, R. W. B. J. A. (2002). Estudios transculturales en comportamiento organizacional y del consumidor. Boletín de Psicología, (76), 77-107.

Berry, J.W., Poortinga, Y. H., Segal, M. H., \& Dasen, P. (2002). Cross-cultural Psychology: Research and applications (2 ${ }^{\mathrm{a}}$ ed.). Cambridge: Cambridge University Press.

Bolsoni-silva, A. T. \& Del Prette, A. (2003). Problemas de comportamento: um panorama da área. Revista Brasileira de Terapia Comportamental e Cognitiva, V(2), 91-103.

Borsa, J. \& Nunes, M. L. (2008). Concordância parental sobre problemas de comportamento infantil através do CBCL. Paidéia (Ribeirão Preto), 18(40), 317-330. https://doi.org/10.1590/S0103-863X2008000200009

Borsa, J. \& Nunes, M. L. (2011a). Prevalence of behavior problems in a sample of school. Aletheia, 34, 1-11.

Borsa, J. \& Nunes, M. L. (2011b). Prevalência de problemas de comportamento em uma amostra de crianças em idade escolar da cidade de Porto Alegre. Aletheia, 34, 1-11.

Borsa, J., Souza, D. S., \& Bandeira, D. R. (2011). Prevalência dos problemas de comportamento em uma amostra de crianças do Rio Grande do Sul. Psicologia: Teoria e Pratica, 13(2), 15-29.

Brewis, A., Schmidt, K., \& Casas, C. A. S. (2003). Cross-cultural study of the childhood developmental trajectory of attention and impulse control. International Journal of Behavioral Development, 27(2), 174-181. https://doi. org/10.1080/0165025024400173

Cunill, R. \& Xavier, C. (2015). Trastorno por déficit de atención con hiperactividad. Med Clin, 144(8), 370-375. https:// doi.org/10.1016/j.medcli.2014.02.025

Dumas, J. E. (2011). Psicopatologia da infância e da adolescência (3ae ed.). Porto Alegre: Artmed.

Egisdóttir, S., Gerstein, L. H., \& Çinarbas, D. C. (2008). Methodological issues in cross-cultural counseling research: equivalence, bias, and translations. The Counseling Psychologist, 36(2), 188-219. https://doi. org/10.1177/0011000007305384

Emerich, D. R., Da Rocha, M. M., Silvares, E. F. D. M., \& Gonçalves, J. D. P. (2012). Diferenças quanto ao gênero entre escolares brasileiros avaliados pelo Inventário de Comportamentos para Crianças e Adolescentes (CBCL/6-18). PSICO, 43(3), 380-387.

Fernández-Molina, M., Valle, J., Fuentes, J., Isabel María Bernedo, I. M., \& Bravo, A. (2011). En Adolescentes Adoptados Españoles. Psicothema, 23(1), 1-6.

Foley, D. L., Rutter, M., Angold, A., Pickles, A., Maes, H. M., Silerg, J. L., \& Eaves, L. J. (2005). Making sense of informant disagreement for overanxious disorder. Journal of Anxiety Disorders, 19, 193-210. https://doi.org/10.1016/j. janxdis.2004.01.006 
Gómez, A., Santelices, M. P., Gómez, D., Rivera, C., \& Farkas, C. (2014). Problemas conductuales en preescolares chilenos: percepción de las madres y del personal educativo. Estudios Pedagógicos, 2, 175-187. https://doi. org/10.4067/S0718-07052014000300011

Hess, A. R. B. \& Falcke, D. (2013). Sintomas internalizantes na adolescência e as relações familiares: uma revisão sistemática da literatura. Psico-USF, 18(2), 263-276. https://doi.org/10.1590/S1413-82712013000200010

Jacob, C., Gross-Lesch, S., Jans, T., Geissler, J., Reif, A., Dempfle, A., \& Lesch, K. P. (2014). Internalizing and externalizing behavior in adult ADHD. ADHD Atten Def Hyp Disord, 6, 101-110. https://doi.org/10.1007/s12402014-0128-Z

Kuja-Halkola, R., Lichtenstein, P., D’Onofrio, B. M., \& Larsson, H. (2015). Codevelopment of ADHD and externalizing behavior from childhood to adulthood. Journal of Child Psychology and Psychiatry and Allied Disciplines, 56(6), 640-647. https://doi.org/10.1111/jcpp.12340

Lampert, T. L. (2002). Avaliação da acurácia diagnóstica da escala de problemas de atenção do Inventário de Comportamentos da Infância e Adolescência (CBCL) para o transtorno de déficit de atenção e hiperatividade. Universidade Federal do Rio Grande do Sul.

López Soler, C., Castro Sáez, M., Alcántara López, M. V., Fernández Fernández, V., López Pina, J. A., López-Soler, C., ... López Pina, J. A. (2009). Prevalencia y características de los síntomas externalizantes en la infancia. Diferencias de género. Psicothema, 21(3), 353-358. Retrieved from http://dialnet.unirioja.es/servlet/articulo? codigo=3024951 \&orden=219384\&info=link\nhttp://dialnet.unirioja.es/servlet/extart?codigo=3024951

López-Soler, C. \& Romero Medina, A. (2013). TDAH y trastornos del comportamiento en la infancia y la adolescencia: clínica, diagnóstico, evaluación y tratamiento (Ediciones). Madrid.

López-Soler, C., Sáez, M. C., López, M. A., Fernández, V. F., \& Pina, J. a L. (2009). Prevalencia y características de los síntomas externalizantes en la infancia. Diferencias de género. Psicothema, 21, 353-358.

Miranda, A., Colomer, C., Berenguer, C., Roselló, R., \& Roselló, B. (2015). Substance use in young adults with ADHD: Comorbidity and symptoms of inattention and hyperactivity/impulsivity. International Journal of Clinical and Health Psychology, 1-9. https://doi.org/10.1016/j.ijchp.2015.09.001

Navarro, M. \& García-Villamisar, D. (2011). Comorbilidad entre el trastorno por déficit de atención con hiperactividad y los trastornos internalizantes. International Journal of Developmental and Educational Psychology, 4(1), 295-304 Retrieved from http://infad.eu/RevistaINFAD/2011/n1/volumen4/INFAD 010423 295-304.pdf

Nolen-Hoeksema, S., Fredrickson, B., Lofus, G., \& Wagenaar, W. (2012). Introdução à psicologia. São Paulo: Cengage Learning.

Ohlmeier, M. D., Peters, K., Te Wildt, B. T., Zedler, M., Ziegenbein, M., Wiese, B., ... Schneider, U. (2008). Comorbidity of alcohol and substance dependence with attention-deficit/hyperactivity disorder (ADHD). Alcohol and Alcoholism (Oxford, Oxfordshire), 43(3), 300-304. https://doi.org/10.1093/alcalc/agn014

Olatundun, I. O. (2009). What is Cross-cultural Research? International Journal of Psychological Studies, 1(2), 82-96. https://doi.org/10.5539/ijps.v1n2p82

Pérez-lópez, J., Cano-giménez, E., \& Agustín, R. (2009). La percepción de problemas conductuales a través de progenitores y educadores de escuelas infantiles. INFAD Revista de Psicología, 1, 133-140.

Pires, T. de O., Passos, C. M. F., Silva, D., \& Assis, S. G. de. (2012). Family environment and attention-defi cit hyperactivity. Rev Saúde Pública.

Polanczyk, G. V., Salum, G. A., Sugaya, L. S., Caye, A., \& Rohde, L. A. (2015). Annual research review: A metaanalysis of the worldwide prevalence of mental disorders in children and adolescents. Journal of Child Psychology and Psychiatry and Allied Disciplines, 56(3), 345-365. https://doi.org/10.1111/jcpp.12381

Possa, M. D. A., Spanemberg, L., \& Guardiola, A. (2005). Comorbidades do transtorno de déficit de atenção e hiperatividade em crianças escolares. Arq Neuropsiquiatr, 63(2-B), 479-483. https://doi.org/10.1590/S0004-282X2005000300021

Rescorla, L., Achenbach, T., Ivanova, M. Y., Harder, V. S., Otten, L., Bilenberg, N., ... Leung, P. W. L. (2011). International Comparisons of Behavioral and Emotional Problems in Preschool Children: Parents 'Reports From 24 Societies. Journal of Clinical Child \& Adolescent Psychology, 40(3), 456-467. https://doi.org/10.1080/15374416.2011.563472

Rescorla, L., Achenbach, T., Ivanova, M., Dumenci, L., Almqvist, F., Bilenberg, N., ... Verhulst, F. (2007). Behavioral and Emotional Problems Reported by Parents of Children Ages 6 to 16 in 31 Societies. Journal of Emotional and Behavioral Disorders, 15, 130-142. https://doi.org/10.1177/10634266070150030101

Rivera, O., Martínez, A., \& Pérez, J. (2005). Comparación de la sintomatología internalizante y externalizante en un grupo de niños y niñas con y sin trastorno de déficit de atención con hiperactividad. Revista Puertorirqueña de Psicología, 16, 27-50.

Roessner, V., Becker, A., Rothenberger, A., Rohde, L., \& Banaschewski, T. (2007). A cross-cultural comparison between samples of Brazilian and German children with ADHD/HD using the Child Behavior Checklist. Eur Arch Psychiatry Clin Neurosci, 257, 352-359. https://doi.org/10.1007/s00406-007-0738-y

Rohde, L. A. (2002). ADHD in Brazil: the DSM-IV criteria in a culturally different population. Journal of the American Academy of Child and Adolescent Psychiatry, 41(9), 1131-1133. https://doi.org/10.1097/00004583-200209000-00014

Rohde, L., Szobot, C., Polanczyk, G., Schmitz, M., Martins, S., \& Tramontina, S. (2005). Attention-deficit/hyperactivity disorder in a diverse culture: Do research and clinical findings support the notion of a cultural construct for the disorder? Biological Psychiatry, 57(11), 1436-1441. https://doi.org/10.1016/j.biopsych.2005.01.042 
Schoemaker, K., Mulder, H., Dekovi, M., Bennett, D. S., Marini, V. A., Berzenski, S. R., ... Engels, R. C. M. E. (2013). Externalizing problems in late childhood as a function of prenatal cocaine exposure and environmental risk, 247(January 2011), 239-247. https://doi.org/10.1177/0165025413490865

Schroeder, V. M., \& Kelley, M. L. (2009). Associations between family environment, parenting practices, and executive functioning of children with and without ADHD. Journal of Child and Family Studies, 18(2), 227-235. https://doi. org/10.1007/s10826-008-9223-0

Skogli, E., Teicher, M. H., Andersen, P., Hovik, K., \& Øie, M. (2013). ADHD in girls and boys - gender differences in co-existing symptoms and executive function measures. BMC Psychiatry, 13(1), 298. https://doi.org/10.1186/1471244X-13-298

Treuting, J. \& Hinshaw, S. (2001). Depression and self-esteem in boys with attention-deficit/hyperactivity disorder: Associations with comorbid aggression and explanatory attributional mechanisms. Journal of Abnormal Child Psychology, 29(1), 23-39. https://doi.org/10.1023/A:1005247412221

Tsiantis, J., Motti-Stefanidi, F., Richardson, C., Schmeck, K., \& Poustka, F. (1994). Psychological problems of school-age German and Greek children a cross-cultural study. European Child and AdolescentPsychiatry, 3(4), 209-219. https:// doi.org/10.1007/BF01978110

Tureck, K., Matson, J. L., May, A., \& Turygin, N. (2013). Externalizing and tantrum behaviours in children with ASD and ADHD compared to children with ADHD. Developmental Neurorehabilitation, 16(February), 52-57. https://doi. org/10.3109/17518423.2012.719245

Valsiner, J. (2012). Fundamentos da psicologia cultural: mundos da mente, mundos da vida. Porto Alegre: Artmed.

Vaquerizo-Madrid, J. (2005). Hiperactividad en el niño preescolar: descripción clínica. Revista de Neurología, 40 (Supl 1), 25-32.

Whitbourne, S. \& Halgin, R. (2015). Psicopatologia: perspectivas clínicas dos transtornos psicológicos. Porto Alegre: AMGH.

World Health Organization. (2014). Health for the World's Adolescents a second chance in the second decade. Geneva.

Autoras:

Ana Flávia Lima Teles da Hora - Doutora pela Universidade Federal do Pará. Docente na Universidade Ceuma.

Concepción López - Doutora, Universidad de Murcia - Espanha.

Petruska Oliveira Baptista - Mestre pela Universidade Federal do Pará.

Simone Souza da Costa Silva - Pós-Doutora, Universidade Federal do Pará.

Endereço para correspondência:

Ana Flávia Lima Teles da Hora

Rua 01, Casa 02 - Solar dos Lusitanos

65065642 - São Luís, MA, Brasil

<anaflaviadahora@hotmail.com>

Recebido em: 04.10.2016

Aceito em: 01.03.2017 